\title{
LETTERS
}

\section{Strategies to support health care providers during the COVID-19 pandemic}

We thank Wu and colleagues for their important commentary regarding potential psychological effects of coronavirus disease 2019 (COVID-19) on health care providers. ${ }^{1}$ Our group of multidisciplinary oncology providers was formed to support oncology health care providers, a group at high risk of burnout. ${ }^{2}$ We outline additional strategies we have developed, which complement this commentary.

The wellness of health care providers is a spectrum, from engagement to burnout; we recognize that individual characteristics, experiences and organizational factors can influence one's position on this spectrum. ${ }^{3}$ Our approach follows 5 key considerations for health care providers during the COVID-19 pandemic, developed by Shanafelt. ${ }^{4}$ At the organizational level, our group advocates to ensure that the first 3 principles ("hear me," "protect me" and "prepare me") are factored into decision-making and communication.

We developed resources aligned with the "support me" and "care for me" categories. First, "CREATE" (Compassion and Resilience Team-building) pairs a psychosocial services professional with clinical managers to offer support and embed low-dose interventions into clinical teams using a coaching and psychological first aid model. Second, we created a toolkit with information on accommodation, grocery delivery, safety, coping and mental health resources. We also implemented the "Buddy Up" program to foster connectedness, by asking health care providers to form groups of twos or threes and check in daily via phone, email or text message. Other interventions include "virtual coffee mornings" to debrief, online exercise programs and virtual meditation lounges led by trained physicians. These provide tailored options for health care providers to achieve connection, mindfulness, movement and meaning.

The COVID-19 pandemic is an unprecedented event that has challenged health care providers' wellness and resilience; however, it provides a unique opportunity to address institutional gaps in dealing with such stressors. We hope that organizations continue "hearing, protecting, preparing, supporting and caring" for health care providers beyond this pandemic.

\section{Michelle B. Nadler MD}

Medical oncology fellow, Department of Medical Oncology and Haematology, Princess Margaret Cancer Centre, Toronto, Canada and University of Toronto, Toronto, Ont.

\section{Aisling Barry MD}

Radiation oncologist, Radiation Medicine Program, Princess Margaret Cancer Centre, Toronto, Canada and University of Toronto, Toronto, Ont.

\section{Tracy Murphy MD}

Hematologist, Department of Medical Oncology and Haematology, Princess Margaret Cancer Centre, Toronto, Canada and University of Toronto, Toronto, Ont.

\section{Rebecca Prince MD}

Medical oncologist, Department of Medical Oncology and Haematology, Princess Margaret Cancer Centre, Toronto, Canada and University of Toronto, Toronto, Ont.

\section{Mary Elliott MD}

Psychiatrist, Department of Supportive Care, Princess Margaret Cancer Centre, Toronto, Canada and University of Toronto, Toronto, Ont.

- Cite as: CMAJ 2020 May 11;192:E522. doi: $10.1503 / \mathrm{cmaj} .75499$

\section{References}

1. Wu PE, Styra R, Gold WL. Mitigating the psychological effects of COVID-19 on health care workers. CMAJ 2020;192:E456-8.

2. Hlubocky FJ, Back AL, Shanafelt TD. Addressing burnout in oncology: why cancer care clinicians are at risk, what individuals can do, and how organizations can respond. Am Soc Clin Oncol Educ Book 2016;35:271-9.

3. Shanafelt TD, Noseworthy JH. Executive leadership and physician well-being: nine organizational strategies to promote engagement and reduce burnout. Mayo Clin Proc 2017;92:129-46.

4. Shanafelt T, Ripp J, Trockel M. Understanding and addressing sources of anxiety among health care professionals during the COVID-19 pandemic. JAMA 2020 Apr. 7. [Epub ahead of print] doi: 10.1001/jama.2020.5893.

Competing interests: None declared. 